Ann. Biol. anim. Bioch. Biophys., I969, 9 (4), 475-481.

\title{
VARIATIONS AVEC L'AGE ET LE SEXE DES TENEURS EN HORMONES FOLLICULO-STIMULANTE (FSH) ET LUTÉINISANTE (LH) DES ANTEHYPOPHYSES DE FETUS DE MOUTON
}

\author{
P. MAULÉN et Marie-Madeleine de REVIERS \\ avec la collaboration technique de Andrée Chevereau \\ Station de Recherches sur la Physiologie de la Reproduction, \\ Centre de Recherches de Tours, 37 - Nouzilly \\ Institut national de la Recherche agronomique
}

\section{SOMMAIRE}

L'activité LH peut être dosée par la méthode de PaRLow dans les hypophyses de fœetus de brebis de 60 à 80 jours ; cette teneur augmente avec l'âge du fotus.

L'activité FSH n'est dosable par la méthode de STEELMaN et PoHLEy qu'à partir de 80 à roo jours mais la concentration est dès cet âge aussi élevée que peu avant la naissance.

Il existe donc des activités FSH et LH dans l'hypophyse de foetus de brebis pendant la période où s'édifient les follicules primordiaux (période ovogénétique proprement dite.) La teneur hypophysaire en LH est identique chez les foetus mâles et femelles, mais celle en FSH n'est élevée que chez la femelle.

\section{INTRODUCTION}

De nombreux faits tendent à montrer qu'une activité gonadotrope hypophysaire débute pendant la vie foetale des Mammifères.

Les études histologiques de 1'hypophyse de fœetus (COOPER, I925; HAMMAR, I925 ; RUMPH et SMITH, I926 ; BRAHMS, I932 ; NELSON, I933 ; KIRMAN, I937) apportent un certain nombre de présomptions sur l'existence d'une activité sécrétoire du parenchyme hypophysaire. Des grains PAS positifs (JosT et GoNSE, I953 ; JosT et TAVERNIER, I958 ; FALIN, I96r) et des granules à ultrastructure caractéristique (DuBOIS et Dumont, I965 et I966) y ont d'ailleurs été mis en évidence. Cependant seuls, jusqu'à maintenant, DuBors et HERLANT (I967) ont différencié dans 1'hypophyse du foetus de Veau des cellules ayant les mêmes affinités tinctoriales que celles considérées comme sécrétant FSH et $\mathrm{LH}$ chez 1'adulte. 
Des implants d'hypophyses de fotus de Porc (Smith et DorTzbach, I929) ou des injections d'extraits hypophysaires de fœtus de Cheval en fin de gestation (HELLBAUM, I935) ont provoqué l'apparition de corps jaunes sur des ovaires de Souris immatures.

De même, la technique de décapitation des fœetus suivie d'injection d'hormones gonadotropes a clairement démontré l'existence d'une relation gonade-hypophyse foetale (JosT, I95I).

Cependant, si l'activité gonadotrope globale ne fait aucun doute, peu de données ont permis de différencier les deux activités FSH ou LH dans 1'hypophyse fœtale.

KARG (I967) n'a dosé l'activité LH qu'au troisième mois de la gestation chez le fœetus de Veau et MELAMPy et al. (I966) se contentent de signaler des traces de LH dans l'hypophyse du fotus de Porc au $80^{\mathbf{e}}$ jour de la gestation et une teneur très élevée au moment de la naissance.

Aucune étude ne permet donc actuellement de se faire une idée sur les activités comparées de FSH et de $\mathrm{L} H$ au cours de la vie embryonnaire ni sur l'influence du sexe sur ces activités. Or les gonades mâles et femelles ont une évolution différente pendant la vie fotale chez la plupart des espèces. Ainsi chez les Ovins, les structures définitives des ovaires : ovogenèse, formation des follicules primordiaux, s'édifient entre le $30^{\mathbf{e}} \mathrm{et}$ le roo jour de la vie fœtale (MAUl,́́ON, I96I) alors que celles des testicules ne se mettent en place qu'après la naissance (CoURoT, I962).

C'est pourquoi nous avons cherché à doser biologiquement les activités FSH et LH dans des hypophyses de fotus de Mouton entre le $3^{\mathbf{e}}$ et le Ioo $^{\mathbf{e}}$ jour de la gestation. Des résultats préliminaires ont été présentés précédemment (MAULÉoN, r967).

\section{MATÉRIEL ET MÉTHODES}

Les hypophyses de fœetus de brebis de race Ile-de-France dont l'âge est connu avec une précision de $\pm \mathrm{I} / 2$ jour sont prélevées à partir du $6 \mathrm{o}^{\mathrm{e}}$ jour de la gestation. Avant ce moment, il est impossible de recueillir la glande sans la léser (tissus très riches en eau). Le prélèvement est fait dans les cinq minutes qui suivent l'abattage de la mère. Les hypophyses sont refroidies immédiatement dans l'acétone à $-15^{\circ}$ et conservées à cette température. Ultérieurement elles sont mises en poudre après avoir été groupées par sexe et par classes d'âge espacées de 20 jours.

Les dosages biologiques des activités LH et FSH sont effectués sur des rattes de 23 jours de la souche Wistar 03-C. R. V. Z. respectivement par les méthodes de Parlow (1958) et de STEELman et POHLEY (1953). Les dosages sont effectués en quatre points et l'analyse statistique réalisée selon EMMENs (I942) et Bliss (1951).

\section{RÉSULTATS}

Les hypophyses sont pesées après dessiccation : le poids moyen à un âge donné est le poids total divisé par le nombre d'hypophyses constituant le pool. Les variations individuelles ne sont donc pas connues. Le poids moyen des hypophyses sèches de fœetus de Mouton augmente avec l'âge entre le $60^{\mathrm{e}}$ jour de la gestation et la mise bas. Il n'existe pas de différence notable entre sexes, quel que soit 1'âge des fœtus (tabl. I).

L'activité $L H$ est dosable par la méthode de ParLow dans les hypophyses de fœtus mâles et femelles à partir de 60 et 80 jours. Une teneur équivalant environ à 


\section{TABLEAU I}

Poids moyen des antéhypophyses de foetus après deshydratation par l'acétone

\begin{tabular}{|c|c|c|}
\hline $\begin{array}{l}\text { Age des foetus } \\
\text { (jours) }\end{array}$ & $\begin{array}{l}\text { Hypophyses des mâles } \\
\text { (mg) }\end{array}$ & $\begin{array}{l}\text { Hypophyses des femelles } \\
\text { (mg) }\end{array}$ \\
\hline $\begin{array}{r}60-79 \\
80-99 \\
100-119 \\
120-140\end{array}$ & $\begin{array}{rr}1,53 & (33) \\
4,37 & (38) \\
9,46 & (30) \\
15,82 & (23)\end{array}$ & $\begin{array}{rr}1,49 & (45) \\
4,32 & (32) \\
10,08 & (39) \\
16,35 & (20)\end{array}$ \\
\hline
\end{tabular}

( ) Nombre de fotus formant le pool d'hypophyses.

TABIEAU 2

Teneur en $L H$ des antéhypophyses de fotus de mouton

\begin{tabular}{|c|c|c|c|c|}
\hline \multirow{2}{*}{$\begin{array}{l}\text { Age des foetus } \\
\text { (jours) }\end{array}$} & \multicolumn{4}{|c|}{$\begin{array}{l}\text { Teneur moyenne des hypophyses de foetus } \\
\text { (en } \mu \mathrm{g} \text { équivalent } \mathrm{LH}-\mathrm{NIH}-\mathrm{S}_{1} \text { par mg de poudre acétonique) }\end{array}$} \\
\hline & \multicolumn{2}{|r|}{ Mâles } & \multicolumn{2}{|c|}{ Femelles } \\
\hline $60 \cdot 79$ & $\begin{array}{l}0,80 \\
1,21\end{array}$ & $\begin{array}{r}(0,39-1,66) \\
\lambda=0,289 \\
(0,61-2,38) \\
\lambda=0,342\end{array}$ & 1,19 & $\begin{array}{l}(0,62-2,25) \\
\lambda=0,342\end{array}$ \\
\hline $80-99$ & $\begin{array}{l}0,68 \\
0,36\end{array}$ & $\begin{array}{c}(0,17-2,64) \\
\lambda=0,365 \\
(0,15-0,80) \\
\lambda=0,271\end{array}$ & 1,11 & $\begin{array}{l}(0,55-2,21) \\
\lambda=0,271\end{array}$ \\
\hline $100-119$ & 6,06 & $\begin{array}{c}(3,01-12,21) \\
\lambda=0,292 \\
(1,81-4,16) \\
\lambda=0,271\end{array}$ & $\begin{array}{r}2,87 \\
0,66 \\
0,69\end{array}$ & $\begin{array}{l}(1,37-6,01) \\
\lambda=0,309 \\
(0,29-1,49) \\
\lambda=0,327 \\
(0,39-1,24) \\
\lambda=0,271\end{array}$ \\
\hline $120-140$ & $\begin{array}{l}6,35 \\
6,06\end{array}$ & $\begin{array}{r}(2,77-14,56) \\
\lambda=0,404 \\
(3,01-12,21) \\
\lambda=0,292\end{array}$ & 5,48 & $\begin{array}{l}(3,42-8,80) \\
\lambda=0,292\end{array}$ \\
\hline
\end{tabular}

$\boldsymbol{\lambda}=$ Index de précision.

Entre parenthèses : intervalles de confiance. 
I $\mu \mathrm{g}$ de $\mathrm{L}_{\mathrm{H}}-\mathrm{NIH}-\mathrm{S}_{\mathbf{1}}$ par milligramme de poids sec existe jusqu'à roo jours. A cet âge elle augmente rapidement chez le mâle, plus progressivement chez la femelle pour atteindre vers la fin de la gestation une valeur équivalant environ à $6 \mu \mathrm{g}$ de LH-NIH-S par milligramme de poids sec dans les hypophyses des fotus des deux sexes (tabl. 2).

$L$ 'activité $F S H$ n'a pu être dosée avant la période de 80 à Ioo jours mais dès ce moment la teneur en FSH des hypophyses de femelles est élevée et n'augmente plus chez les fœtus plus âgés. Par contre, chez les fœetus mâles, quel que soit leur âge, 1'activité FSH est trop faible pour être dosée par cette méthode (tabl. 3).

\section{TABLEAU 3}

Teneur en FSH des antéhypophyses de foetus de mouton

\begin{tabular}{|c|c|c|c|}
\hline \multirow{2}{*}{$\begin{array}{l}\text { Age des foetus } \\
\text { (jours) }\end{array}$} & \multicolumn{3}{|c|}{$\begin{array}{l}\text { Teneur moyenne des hypophyses de foetus } \\
\text { (en } \mu \mathrm{g} \text { équivalent } \mathrm{FSH}-\mathrm{NIH}-\mathrm{S}_{3} \text { par mg de poudre acétonique) }\end{array}$} \\
\hline & Mâles & & emelles \\
\hline & & & $\begin{array}{l}(2,43-5,00) \\
\lambda=0,131\end{array}$ \\
\hline $100-119$ & $<1,2$ & 4,44 & $\begin{array}{l}(3,30-5,96) \\
\lambda=0,172\end{array}$ \\
\hline $120-140$ & $<1,2$ & 4,95 & $\begin{array}{l}(3,17-7,93) \\
\lambda=0,172\end{array}$ \\
\hline
\end{tabular}

$\lambda=$ Index de précision du dosage.

Entre parenthèses : intervalles de confiance.

\section{DISCUSSION}

Les dosages biologiques actuellement à notre disposition permettent de déterminer l'activité $L H$ dans les hypophyses de foetus de mouton âgés de 60 à 80 jours, c'est-à-dire dès que les hypophyses peuvent être facilement prélevées.

Nous n'avons pu doser l'activité FSH avant la période 80-roo jours chez les fœtus. En effet l'injection de ro mg de poudre hypophysaire de foetus de cet âge a donné une réponse trop faible par rapport au standard, pour que la teneur soit calcu. lable.

D'autres techniques plus sensibles permettraient sans doute de la doser avant 60 jours. Il en est de même pour l'activité $L H$ puisque sa présence a pu être révélée par la technique d'immunofluorescence dans les cellules hypophysaires de fotus cle Mouton âgés de 49 jours (Dubors et MaULÉon, I969).

En dépit de leurs limites, les méthodes de dosages biologiques nous ont permis de dégager deux faits intéressants : 


\section{Activités FSH et LH pendant la période ovogénétique}

Lorsque se déroulent les multiplications ovogoniales et la prophase méiotique dans les ovocytes, c'est-à-dire avant le Ioo jour de la gestation, 1'hypophyse de fœetus femelle de mouton contient les deux hormones gonadotropes FSH et L.H. Certes la présence d'un contenu hypophysaire riche en hormones n'est pas la preuve d'une libération de celles-ci dans le sang du fœetus. S'il n'existe pas de démonstration parfaite, un certain nombre de faits sont cependant en faveur d'un fonctionnement du complexe hypothalamo-hypophysaire pendant la deuxième moitié de la vie embryonnaire : présence de FSH-RF dans l'éminence médiane du fœtus de 20 jours chez le Rat (CORBIN et DANIELS, I967), activités neuro-sécrétoires révélées par la technique de l'aldéhyde fuchsine de Gomori dans l'hypothalamus de l'Armadillo (WURSTER et BenIRschke, I964), de la Vache (KIVAlio et TaIANTI, I957), de l'Homme (RINNE et al., Ig62) et du Poulet (Mosier, I955).

\section{Différences de concentration hypophysaire en FSH entre les foetus des deux sexes}

La richesse en FSH de l'hypophyse de fœtus ovin femelle s'oppose à la pauvreté de celle du fœtus mâle, alors qu'il n'y a pas de différence entre les deux sexes dans le contenu hypophysaire en $\mathrm{LH}$ chez le fotus de Mouton, chez le fotus de Veau (KARG, I967) ni chez le fœtus humain (RICE et al., I968).

Une telle comparaison est actuellement impossible chez les Ovins après la naissance car on sait seulement que chez le mâle la teneur en LH augmente de $5 \mu \mathrm{g}$ par milligramme d'antéhypophyse à $25 \mu \mathrm{g}$ environ au moment où débute la spermatogenèse, tandis que celle de FSH est à peu près constante jusqu'à la puberté mais à un niveau plus élevé que chez le fœtus (SKInNER et al., I968; CoUROT, non publié). Aucun dosage n'existe chez 1'Agnelle. Nous savons seulement que chez la Génisse, peu après la naissance, la teneur en FSH est 2 à 3 fois plus élevée que chez l'adulte cyclique (DEsjardins et HaFs, I968). Comme l'Agnelle et la Génisse naissent à un stade physiologique comparable, nous pouvons supposer que l'hypophyse del'Agnelle est riche en FSH et que la différence trouvée entre les fœtus des deux sexes se poursuit après la naissance pour disparaitre au moment de la diminution en FSH et LH qui accompagne la puberté.

Reçu pour publication en mai 1969.

\section{REMERCIEMENTS}

Nous remercions le Service Endocrinology Study Section du National Institute of Heallh qui nous a aimablement fourni les hormones de référence LH-NIH-S1 et FSH-NIH-Sa? 


\title{
SUMMARY
}

\author{
CHANGES IN CONCENTRATION OF SHEEP FATUS \\ ANTERIOR PITUITARY FSH AND LH WITH AGE AND SEX
}

LH activity can be estimated in the pituitary gland of male and female sheep fœtuses from day 6o-8o of pregnancy by means of PARLow's method.

FSH activity can only be estimated in female fotuses, from day 80-100, by means of STEELMAN and PoHLEY's method. Male FSH remains in subthreshold amounts throughout pregnancy.

Our results give further evidence that there is a gonadotrophic activity during ovogenesis.

There was no sex difference in LH contents, like in Man and Cow fotus, whereas FSH content was much higher in the female.

LH activity increases until birth, whereas female FSH activity remains at a rather high rate throughout fœtal life.

\section{RÉFÉRENCES BIBLIOGRAPHIQUES}

Bu.jss C. I., I952. The statistics of binassay with special reference to the vitamins. Acad. Press. New York. BraHMS S., 1932. The development of the hypophysis of the cat (Felis domestica). Am. J. Anat., 50, 251271.

COOPER E. R., 1925. The histology of the more important human endocrine organs at various ages. New York Oxford University Press.

Corbin A., DANIELS E. L., 1967. Changes in concentration of female rat pituitary FSH and stalk-median emminence follicle stimulating hormone releasing factor with age. Neuroendocrinology, 2, 304-314.,

Couror M., 1962. Developpement du testicule chez l'Agneau. Établissement de la spermatogenèse. Ann. Biol. anim. Bioch. Biophys., 2, 25-4I.

DESJARDINS C., HAFS H. D., 1968. Levels of pituitary FSH and LH in heifers from birth through puberty J. Anim. Sci., 87, 472-477.

Dubors P., Dumont L., 1965. Observations en microscopie électronique du lobe antérieur de l'hypophyse embryonnaire humaine au troisième mois de la vie intrautérine. C. R. Soc. Biol., 159, 1574.

Dubois P., Dumont L., Ig66. Nouvelles observations au microscope électronique sur l'antéhypophyse humaine du troisième au cinquième mois du développement embryonnaire. C. R. Soc. Biol., 160, aro5.

Dubois M.-P., HERLANT M., I968. Caractères cytologiques des cellules gonadotropes, thyréotropes, corticotropes, somatotropes et des cellules à prolactine présentes dans le lobe antérieur de l'hypophyse des bovins. Ann. Biol. anim. Bioch. Biophys., 8, 5-26.

Dubors M., Maulton P., r969. Mise en évidence par immuno fluorescence des cellules à activité gonadotrope LH dans l'hypophyse du fotus de mouton $C$. R. Acad. Sci, Paris 269, 219-222.

Emmens C. W., 1948. Principles of biological assay. Chapman and Hall, London.

FaLiN L. I., I96r. The develooment of human hypophysis and differentiation of cells of its anterior lobe during embryonnic life. Acta Anat., 44, I88-205.

HAMMAR J. A., I925. A quelle époque de la vie foetale de l'homme apparaissent les premiers signes d'activité glandulaire Usppsala Lâch. För. Förk., 30, 1055.

HELlbaUm A. A., 1935. The gonad-stimulating activity of pituitary glands from horses of different ages and sex types. Anat. Rec., 68, I47-I57.

Jost A., I95I. Recherches sur la différenciation sexuelle du Lapin. IV. Organogenèse sexuelle masculine après décapitation du foetus. Arch. Anat. Microsc. Morph. exp., 40, 247-267.

JOST A., GoNSE P., r953. Application de la méthode à l'acide periodique Schiff (Mac Manus) à l'étude histophysiologique de l'hypophyse foetale du Lapin. Arch. Anat. Microsc. Morph. exp., 42, 243-27 I.

Jost A., Tavernier R., 1956. Application de la méthode à l'acide periodique Schiff (Mac Manus) à l'étude de l'hypophyse foetale du Rat. C. R. Acad. Sc., Paris, 243, 1353-1356.

KARG von H., 1967. Untersuchungen über das gonadotrope hypophysen-hormone LH (ICSH) während der Entwicklung beim Rind. Z. Zuchthygiene, 2, I2-19.

KIRMAN H., 1937. Cytological study of the anterior hypophysis of the guinea-pig and a statistical analysis of the cell types. Am. J. Anat., 61, 233-287.

KIVALO E., TALANTI S., 1957. The fotal development of the hypothalamic-hypophysial neurosecretory system of the cow embryo. Acta Endocrinol., 26, 47 I-476. 
Mauléon P., Ig6I. Déroulement de l'ovogenèse comparée chez différents mammifères domestiques. IV Congr. int. Reprod. anim. Insém. artif., La Haye, 2, 348-354.

Mauléon P., r967. Différenciation et évolution des cellules sexuelles. La lignée femelle. Cinétique de l'ovogenèse chez les Mammifères. Arch. Anat. Microsc. Morph. exp., 56, suppl. 3-4, I25-150.

Melampy R. M., Henricks D. M., Anderson L. L., Chen C. L., Schultz J. R., ig66. Pituitary follicle stimulating hormone and luteinizing hormone concentrations in pregnant and lactating pigs. Endocrinology, 78, 80I-804.

Mosier H. D., 1955. The development of the hypothalamoneurohypophysial secretory system in the chick embryo. Endocrinology, 57, 661-669.

NeLson W. O., 1933. Studies on the anterior hypophysis. I. The development of the hypophysis ia the pig (sus scrofa). II. The cytological differentiation in the anterior hypophysis of the fetal piy. Am.J.Annt., 52, 308-332.

Parlow A. F., I958. A rapid bioassay for LH and factors stimulating LH secretion. Fed. Proc., 17, 402.

Rice B. F., Ponthier R., Sternberg W., 1968. Luteinizing hormone and growth hormona activity of the human fetal pituitary. J. Clin. Endocr. Metab., 23, 1071-1072.

Rinne U. K., Kivalo R., Talanti S., I962. Maturation of human hypothalamic neurosecretion. Biol. Neonatorum., 4, 35I-364.

Rumph P., Smith P. E., I926. The first occurence of secretory products and of a specific structural differentiation in the thyroid and anterior pituitary during the development of the pig foetus. Anat.Rec., 33, 289-298.

Skinner J. D., Booth W. D., Rowson L. E. A., Karg H., r968. The post-natal development of the reproductive tract of the Suffolk ram, and changes in the gonadotrophin content of the pituitary. J.Reprod. Fert., 16, 463-477.

Smith P. E., DoRTzBach C., I929. The first appearance in the anterior pituitary of the developing pig fotus of detectable amounts of the hormones stimulating ovarian maturity and general body growth. Anat. Rec., 43, 277-297.

Steelman S. L., Pohley F. M., 1953. Assay of the follicle stimulating hormone based on the augmentation with human chorionic gonadotrophin. Endocrinology, 43, 604-6I6.

Wurster D. H., Benirschke K., ig64. Development of the hypothalamo-hypophysial neurosecretory system in the fetal Armadillo (Dasypus novemcinctus) with notes on rabbit, cat and dog. Gen. Comp. Endocr., 4, 433-441. 